\title{
Combination of Wishart Test Statistics and Loewner Order for Change Detection in Quad/Full and Dual Polarization SAR Data.
}

\author{
Nielsen, Allan Aasbjerg; Skriver, Henning; Conradsen, Knut
}

Published in:

Proceedings of IEEE International Geoscience and Remote Sensing Symposium

Link to article, DOI:

10.1109/IGARSS47720.2021.9554680

Publication date:

2021

Document Version

Peer reviewed version

Link back to DTU Orbit

Citation (APA):

Nielsen, A. A., Skriver, H., \& Conradsen, K. (2021). Combination of Wishart Test Statistics and Loewner Order for Change Detection in Quad/Full and Dual Polarization SAR Data. In Proceedings of IEEE International Geoscience and Remote Sensing Symposium IEEE. https://doi.org/10.1109/IGARSS47720.2021.9554680

\section{General rights}

Copyright and moral rights for the publications made accessible in the public portal are retained by the authors and/or other copyright owners and it is a condition of accessing publications that users recognise and abide by the legal requirements associated with these rights.

- Users may download and print one copy of any publication from the public portal for the purpose of private study or research.

- You may not further distribute the material or use it for any profit-making activity or commercial gain

- You may freely distribute the URL identifying the publication in the public portal 


\title{
COMBINATION OF WISHART TEST STATISTICS AND LOEWNER ORDER FOR CHANGE DETECTION IN QUAD/FULL AND DUAL POLARIZATION SAR DATA
}

\author{
Allan A. Nielsen ${ }^{1 *}$, Henning Skriver ${ }^{2}$, and Knut Conradsen ${ }^{1}$ \\ ${ }^{1}$ DTU Compute - Applied Mathematics and Computer Science \\ ${ }^{2}$ DTU Space - National Space Institute \\ Technical University of Denmark, DK-2800 Kgs. Lyngby, Denmark
}

\begin{abstract}
We use the combined Wishart-Loewner method to successfully detect change and direction of change in truly multitemporal, multilooked quad/full polarization synthetic aperture radar image data in the covariance matrix representation. Based on in situ data interpretations of the obtained results are given for three selected fields. Histograms of the Wishart test statistics in a wooded no-change area shows good agreement with the theoretical distributions.
\end{abstract}

\section{INTRODUCTION}

This paper uses a method based on the complex Wishart distribution described in [1,2] for change detection in truly multi-temporal, multilook, quad/full polarization synthetic aperture radar (SAR) data in the covariance matrix representation. Many authors have worked with bitemporal change detection in such data, see for example [3-7].

We also use the Löwner (or Loewner) order to determine whether radar signal as measured by the definiteness of the difference of covariance matrices has increased or decreased over time, i.e., to determine the direction of change [8-10].

\section{TEST STATISTICS AND THEIR DISTRIBUTIONS}

Below we very briefly recapitulate the main results from [1] on the test statistics $Q$ and $R_{j}$, and the corresponding probabilities for finding smaller values of $-2 \rho \ln Q$ and $-2 \rho_{j} \ln R_{j}$ relating to change detection in a series of $k \geq 2$ covariance matrix representation multilook SAR data with all (including the off-diagonal) elements in the covariance matrix, see [11].

The average covariance matrix for multilook polarimetric SAR is defined as [11]

$$
\langle\boldsymbol{C}\rangle=\left[\begin{array}{lll}
\left\langle S_{h h} S_{h h}^{*}\right\rangle & \left\langle S_{h h} S_{h v}^{*}\right\rangle & \left\langle S_{h h} S_{v v}^{*}\right\rangle \\
\left\langle S_{h v} S_{h h}^{*}\right\rangle & \left\langle S_{h v} S_{h v}^{*}\right\rangle & \left\langle S_{h v} S_{v v}^{*}\right\rangle \\
\left\langle S_{v v} S_{h h}^{*}\right\rangle & \left\langle S_{v v} S_{h v}^{*}\right\rangle & \left\langle S_{v v} S_{v v}^{*}\right\rangle
\end{array}\right]
$$

where $\langle\cdot\rangle$ denotes ensemble averaging and ${ }^{*}$ denotes complex conjugation. $S_{r t}$ denotes the complex scattering amplitude for receive and transmit polarization $(r, t \in\{h, v\}$ for horizontal and vertical polarization). $\langle\boldsymbol{C}\rangle$ is Hermitian also known as self-adjoint, i.e., it is equal to its own conjugate transpose.

\subsection{Test for equality of several complex covariance matrices}

For the logarithm of the likelihood ratio test statistic $Q$ for testing whether a series of $k \geq 2$ complex variance-covariance matrices $\boldsymbol{\Sigma}_{i}$

\footnotetext{
*alan@dtu.dk, https://people.compute.dtu.dk/alan
}

are equal, i.e., $\boldsymbol{\Sigma}_{1}=\boldsymbol{\Sigma}_{2}=\cdots=\boldsymbol{\Sigma}_{k}$ we get (for the real case see [12]; for the case with two complex matrices see [3,13])

$$
\ln Q=n\left\{p k \ln k+\sum_{i=1}^{k} \ln \left|\boldsymbol{X}_{i}\right|-k \ln \left|\sum_{i=1}^{k} \boldsymbol{X}_{i}\right|\right\} .
$$

Here $|\cdot|$ denotes the determinant, $n$ is the equivalent number of looks, the $\boldsymbol{X}_{i}=n \hat{\boldsymbol{\Sigma}}_{i}=n\langle\boldsymbol{C}\rangle_{i}$ (and the $\boldsymbol{\Sigma}_{i}$ ) are $p$ by $p(p=3$ for full pol data, $p=2$ for dual pol data, and $p=1$ for single channel power data), and the $\boldsymbol{X}_{i}$ follow the complex Wishart distribution, $\boldsymbol{X}_{i} \sim W_{C}\left(p, n, \boldsymbol{\Sigma}_{i}\right)$.

Because $Q$ tests for equality in all matrices simultaneously, it is termed an omnibus test statistic.

For full and dual pol data ( $p=3$ and $p=2$, respectively) with all off-diagonal elements in the covariance matrix and with

$$
\begin{aligned}
f & =(k-1) p^{2} \\
\rho & =1-\frac{p\left(2 p^{2}-1\right)}{6 f}\left(\frac{k}{n}-\frac{1}{n k}\right) \\
\omega_{2} & =-\frac{f}{4}\left(1-\frac{1}{\rho}\right)^{2}+\frac{p^{2}\left(p^{2}-1\right)}{24 \rho^{2}}\left(\frac{k}{n^{2}}-\frac{1}{n^{2} k^{2}}\right)
\end{aligned}
$$

the probability of finding a smaller value of $-2 \rho \ln Q$ is

$$
\begin{aligned}
& P\{-2 \rho \ln Q \leq z\} \simeq P\left\{\chi^{2}(f) \leq z\right\}+ \\
& \omega_{2}\left[P\left\{\chi^{2}(f+4) \leq z\right\}-P\left\{\chi^{2}(f) \leq z\right\}\right],
\end{aligned}
$$

where $z=-2 \rho \ln q$ and $q$ is a particular realization (the observed value) of the stochastic variable $Q$.

\subsection{Test for equality of first $j \leq k$ matrices}

If the above $-2 \rho \ln Q$ test statistic shows that we cannot reject the hypothesis $\boldsymbol{\Sigma}_{1}=\boldsymbol{\Sigma}_{2}=\cdots=\boldsymbol{\Sigma}_{k}$, there is no change over the time span covered by the data. If we reject the hypothesis, there is change at some time point. To find that time point, we test whether the first $j(1<j \leq k)$ complex variance-covariance matrices $\boldsymbol{\Sigma}_{i}$ are equal, i.e., given that $\boldsymbol{\Sigma}_{1}=\boldsymbol{\Sigma}_{2}=\cdots=\boldsymbol{\Sigma}_{j-1}$, then the logarithm of the likelihood ratio test statistic $R_{j}$ for testing $\boldsymbol{\Sigma}_{j}=\boldsymbol{\Sigma}_{1}$ is

$$
\begin{aligned}
\ln R_{j}= & n\{p(j \ln j-(j-1) \ln (j-1))+ \\
& \left.(j-1) \ln \left|\sum_{i=1}^{j-1} \boldsymbol{X}_{i}\right|+\ln \left|\boldsymbol{X}_{j}\right|-j \ln \left|\sum_{i=1}^{j} \boldsymbol{X}_{i}\right|\right\} .
\end{aligned}
$$

Furthermore, the $R_{j}$ constitute a factorization $Q=\prod_{j=2}^{k} R_{j}$ or

$$
\ln Q=\sum_{j=2}^{k} \ln R_{j} .
$$



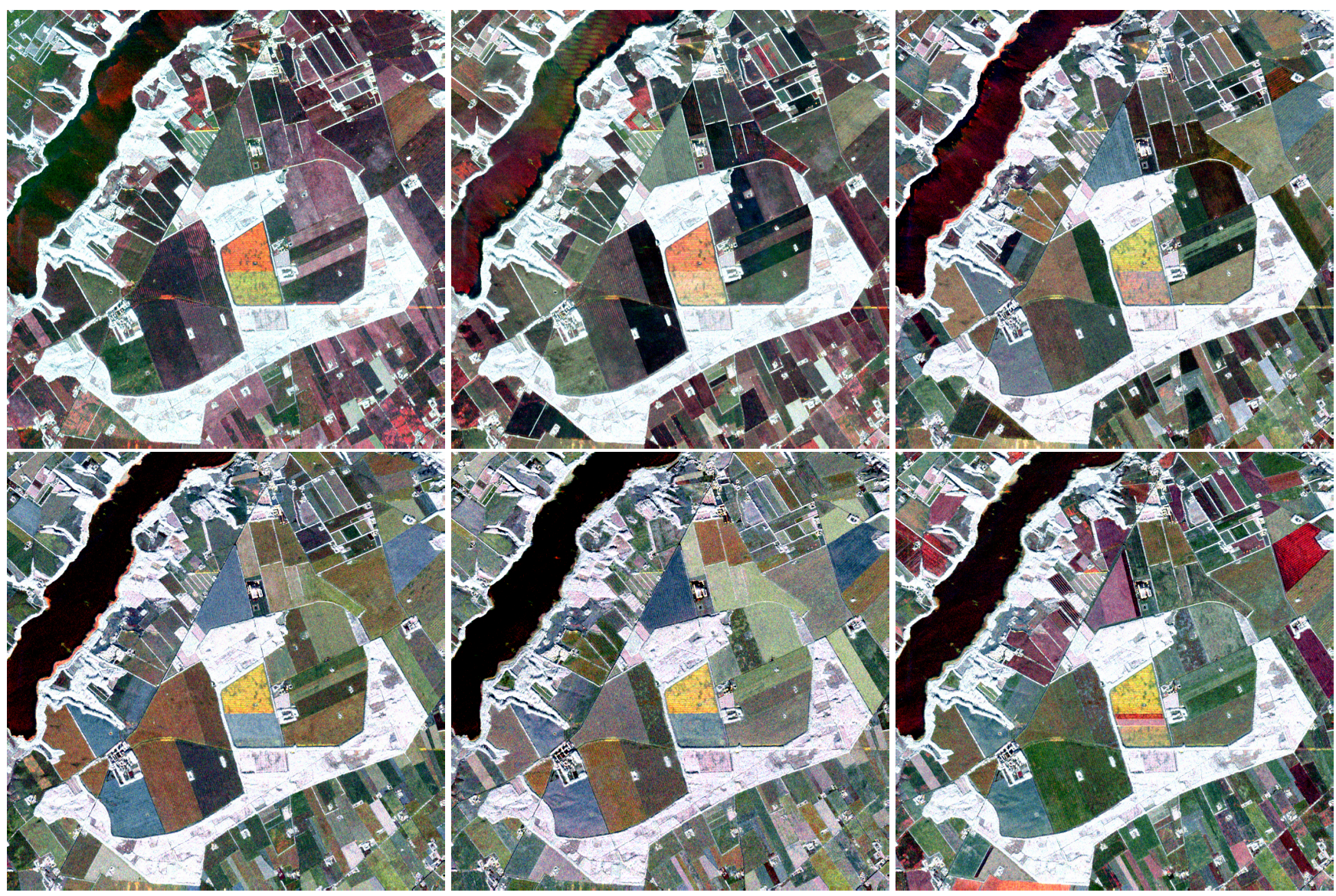

Fig. 1. EMISAR L-band monthly data March-August covering an agricultural region near Foulum, Denmark, logarithms of eigenvalues as RGB ( $\ln \lambda_{1}$ is red, $\ln \lambda_{2} \leq \ln \lambda_{1}$ is green, and $\ln \lambda_{3} \leq \ln \lambda_{2}$ is blue; same stretch to all $\ln \lambda_{1}$, same (but different) stretch to all $\ln \lambda_{2}$, etc.

Change may occur at more than one time point. Hence we establish what we call an omnibus change path for each pixel, see $[1,2]$.

For full and dual pol data ( $p=3$ and $p=2$, respectively) with all off-diagonal elements in the covariance matrix and with

$$
\begin{aligned}
g & =p^{2} \\
\rho_{j} & =1-\frac{2 p^{2}-1}{6 p n}\left(1+\frac{1}{j(j-1)}\right) \\
\omega_{2 j} & =-\frac{p^{2}}{4}\left(1-\frac{1}{\rho_{j}}\right)^{2}+\frac{p^{2}\left(p^{2}-1\right)}{24 n^{2} \rho_{j}^{2}}\left(1+\frac{2 j-1}{j^{2}(j-1)^{2}}\right)
\end{aligned}
$$

the probability of finding a smaller value of $-2 \rho_{j} \ln R_{j}$ is

$$
\begin{gathered}
P\left\{-2 \rho_{j} \ln R_{j} \leq z\right\} \simeq P\left\{\chi^{2}(g) \leq z\right\}+ \\
\omega_{2 j}\left[P\left\{\chi^{2}(g+4) \leq z\right\}-P\left\{\chi^{2}(g) \leq z\right\}\right],
\end{gathered}
$$

where $z=-2 \rho_{j} \ln r_{j}$ and $r_{j}$ is a particular realization (the observed value) of the stochastic variable $R_{j}$.

\subsection{The Loewner order}

For scalar quantities it is easy to establish whether one quantity is larger than another, for example, we can check whether the difference between them is positive, negative or zero. For matrices this is a more complicated matter. The Loewner order provides a partial ordering of matrices [8-10]. Here in our context it gives a direction of change: does the radar response $\boldsymbol{X}_{1}$ at time point one and $\boldsymbol{X}_{2}$ at time point two increase or decrease (or does it possibly change structure or nature) between the two time points? To establish the Loewner order we calculate the definiteness of the difference $\boldsymbol{X}_{1}-\boldsymbol{X}_{2}$. If $\boldsymbol{X}_{1}-\boldsymbol{X}_{2}$ is positive definite, we write $\boldsymbol{X}_{2}<_{L} \boldsymbol{X}_{1}$, if $\boldsymbol{X}_{1}-\boldsymbol{X}_{2}$ is negative definite, we write $\boldsymbol{X}_{1}<_{L} \boldsymbol{X}_{2}$.

\section{CASE STUDY ON EMISAR DATA}

The EMISAR $[14,15]$ L-band data used (which are also reported on in $[1,2])$ are acquired on $t_{1}=21$ March, $t_{2}=17$ April, $t_{3}=20$ May, $t_{4}=16$ June, $t_{5}=15 \mathrm{July}$, and $t_{6}=16$ August. The 13-look images are 1024 rows by 1024 columns 5m pixels, see [1] for more details. Descendingly ordered eigenvalues of the data are shown as RGB in Figure 1. Figure 2 shows the results of pairwise consecutive test for change: positive definite matrix difference in red (i.e., $\boldsymbol{X}_{t_{i+1}}<_{L} \boldsymbol{X}_{t_{i}}$ ), negative definite matrix difference in green (i.e., $\boldsymbol{X}_{t_{i}}<_{L} \boldsymbol{X}_{t_{i+1}}$ ), indefinite matrix difference in yellow, all where $P$-values, i.e., the change probabilities in the Wishart based test are larger than $99 \%$. Red regions exhibit significant change and have stronger backscatter at $t_{i}$, green regions exhibit significant change and have stronger backscatter at $t_{i+1}$, and yellow regions exhibit significant change with a nature so that the Loewner order cannot determine which backscatter is stronger. Hence, the Loewner order gives a measure of direction of detected change. Gray scale regions exhibit no 

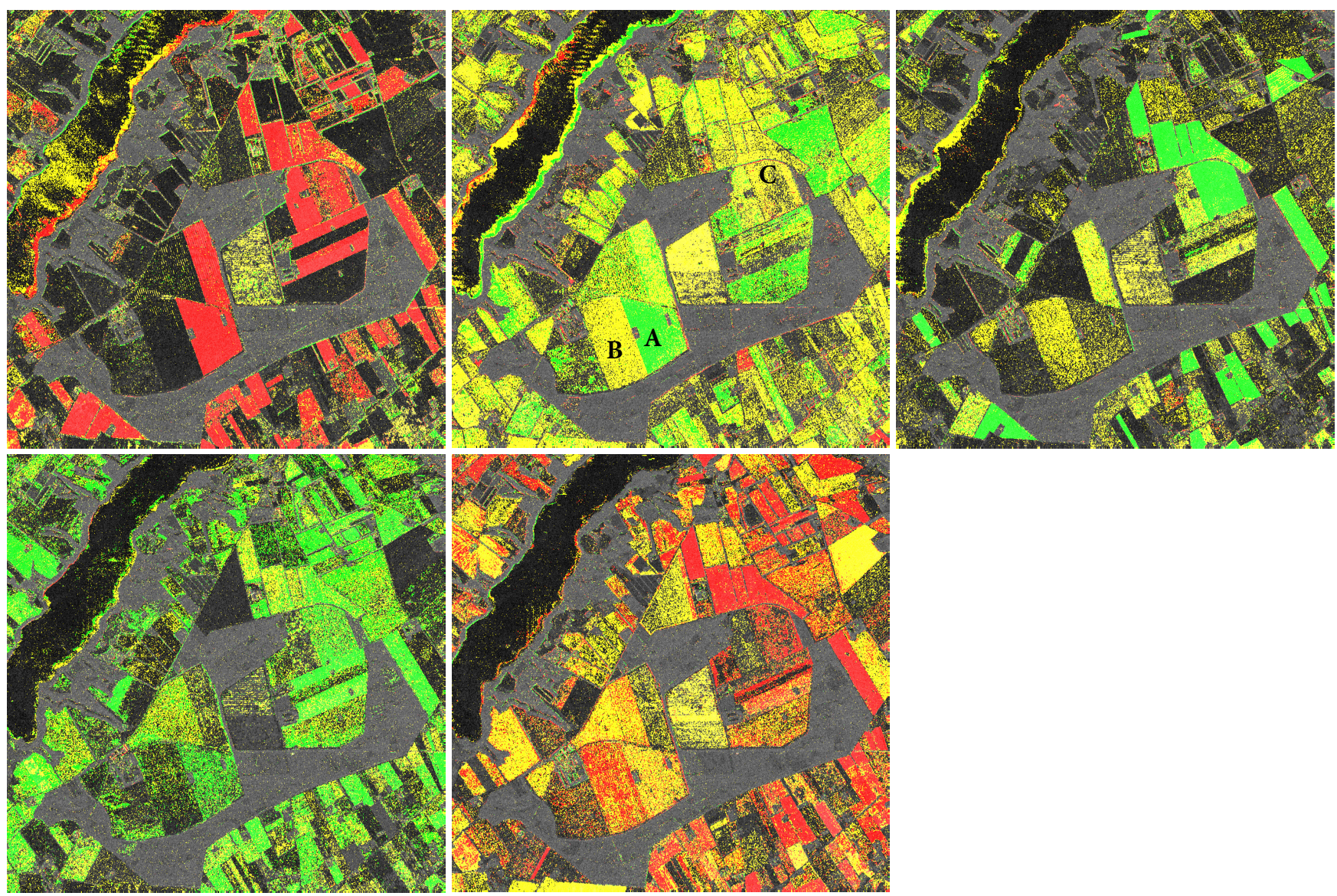

Fig. 2. Pairwise consecutive tests (row-wise March-April, April-May, May-June, June-July, July-August), $P$-values for finding smaller values of $-2 \rho_{2} \ln R_{2}$, i.e., change probabilities thresholded at $99 \%$ and $\boldsymbol{X}_{t_{i}}-\boldsymbol{X}_{t_{i+1}}$ positive definite (red), $P>99 \%$ and $\boldsymbol{X}_{t_{i}}-\boldsymbol{X}_{t_{i+1}}$ negative definite (green), $P>99 \%$ and $\boldsymbol{X}_{t_{i}}-\boldsymbol{X}_{t_{i+1}}$ indefinite (yellow). Red regions have stronger backscatter at time point $t_{i}$, green regions have stronger backscatter at time point $t_{i+1}$. Yellow means significant change with indefinite matrix difference. The gray scale no-change background is the temporal average of $\left\langle S_{v v} S_{v v}^{*}\right\rangle$ of all six images. Note the three fields marked A (spring barley), B (winter wheat) and C (peas) in the top-center image.

significant change. From March to April a number of fields are dominated by a change from a strong backscatter in March to a weaker backscatter in April. This situation corresponds to spring crop fields that change from plowed soil (rough surface) to sowed soil (smoother surface), e.g., the VV-backscatter for field A (spring barley) changes from $-16.4 \mathrm{~dB}$ to $-25.3 \mathrm{~dB}$. From April to May many of the fields with change are represented by indefinite matrix differences (yellow). This corresponds to a change in scattering mechanism with smaller changes in the backscatter coefficients. In this case, it corresponds to a change from surface scattering or attenuated surface scattering (through vegetation for the winter crops) to double bounce scattering, where the vegetation has grown sufficiently in May to scatter back the forward scattering component from the smooth soil. For field $\mathrm{B}$ (winter wheat), for instance, the VV-backscatter is $-18.2 \mathrm{~dB}$ and $-18.3 \mathrm{~dB}$ in April and May, respectively, where the HH-backscatter is $-23.0 \mathrm{~dB}$ and $-17.9 \mathrm{~dB}$. The phase difference between $\mathrm{HH}$ and VV change from $35^{\circ}$ to $-74^{\circ}$. These results indicate the abovementioned change from surface scattering to double bounce scattering. From May to June and from June to July a number of fields show negative definite matrix differences, and the reason is the increased backscatter from volume scattering from a higher and denser vege- tation. For field C (peas) the development stage from May to July changes from leaf development (12 cm height), over emergence (42 $\mathrm{cm}$ height), to development of fruit $(69 \mathrm{~cm}$ height). For instance, the cross-polarized backscatter changes from $-34.8 \mathrm{~dB}$, over $-24.8 \mathrm{~dB}$, to $-18.3 \mathrm{~dB}$, and the entropy changes from 0.53 , over 0.76 , to 0.86 . These results clearly show an increase in the volume scattering from May to July. Finally, the positive definite matrix difference seen between July and August is caused by a decrease in backscatter due to either harvesting or drying out of the crops, in both cases changing the scattering from volume scattering to (attenuated) surface scattering.

Figure 3 shows sample and theoretical distributions for $-2 \rho \ln Q$ (top row plots) and $-2 \rho_{j} \ln R_{j}$ for a small wooded no change area just below field B. Judged visually, the sample distributions fit the theoretical ones nicely.

The following table shows mean values of $-2 \rho \ln Q$ (top row) and $-2 \rho_{j} \ln R_{j}$ for the small wooded no change region just below field $\mathrm{B}$. The first column with numbers shows results for testing $t_{1}=$ $t_{2}=\cdots=t_{6}$, the second column with numbers shows results for testing $t_{2}=\cdots=t_{6}$ (omitting $t_{1}$ ), the third column with numbers shows results for testing $t_{3}=\cdots=t_{6}$ (omitting $t_{1}$ and $t_{2}$ ), etc. Ideal values are the numbers of degrees of freedom. 


\begin{tabular}{|l|rrrrr|}
\hline \hline Omnibus & 51.6318 & 39.3011 & 29.7337 & 20.2582 & 9.8528 \\
$t_{1}=t_{2}$ & 10.7501 & & & & \\
$t_{2}=t_{3}$ & 8.6506 & 7.4819 & & & \\
$t_{3}=t_{4}$ & 10.6303 & 10.6011 & 9.2634 & & \\
$t_{4}=t_{5}$ & 10.7958 & 10.5534 & 9.8638 & 9.7539 & \\
$t_{5}=t_{6}$ & 10.7948 & 10.7335 & 10.6226 & 10.5108 & 9.8528 \\
\hline \hline
\end{tabular}

The following table shows mean values of $1-P\{-2 \rho \ln Q \leq z\}$ (top row) and $1-P\left\{-2 \rho_{j} \ln R_{j} \leq z\right\}$ for the small wooded no change region just below field $B$. The ideal value is $1 / 2$ for all entries.

\begin{tabular}{|l|lllll|}
\hline \hline Omnibus & 0.3360 & 0.4092 & 0.4087 & 0.4024 & 0.4425 \\
$t_{1}=t_{2}$ & 0.3933 & & & & \\
$t_{2}=t_{3}$ & 0.5333 & 0.6149 & & & \\
$t_{3}=t_{4}$ & 0.4054 & 0.4039 & 0.4830 & & \\
$t_{4}=t_{5}$ & 0.4067 & 0.4138 & 0.4461 & 0.4469 & \\
$t_{5}=t_{6}$ & 0.4088 & 0.4072 & 0.4097 & 0.4128 & 0.4425 \\
\hline \hline
\end{tabular}

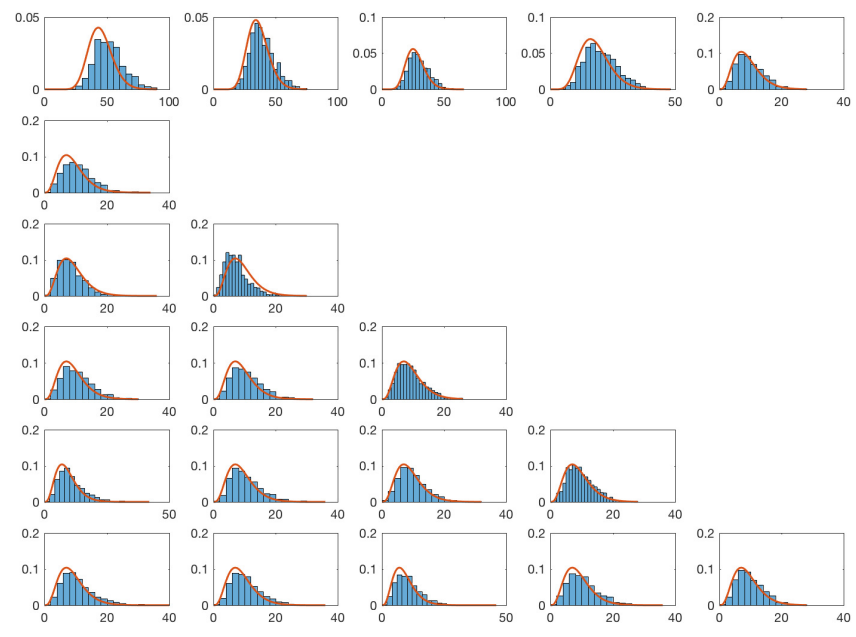

Fig. 3. Sample and theoretical distributions for $-2 \rho \ln Q$ (top row plots) and $-2 \rho_{j} \ln R_{j}$ for a small wooded no change area just below field B. Judged visually, the sample distributions fit the theoretical ones nicely.

\section{CONCLUSIONS}

We have used a change detection scheme for polarimetric SAR data based on a combination of the Wishart detector with an associated p-value and the Loewner order. The Loewner order determines the direction of significant change as detected by the Wishart test statistic. This direction is here determined by the definiteness of the pairwise consecutive matrix differences.

In an example with EMISAR quad/full polarization data, change and direction of change are successfully detected by the combined Wishart-Loewner detector and interpreted for three selected fields.

Plots of sample and theoretical distributions of the test statistics over a no change region show satisfactory agreement.

The methods described here can be used in the analysis of other SAR data also, for example data from Sentinel-1, Radarsat-2, TerraSAR-X, COSMO-SkyMed and GaoFen-3.

Matlab code to perform the above analyses is available on Allan Nielsen's homepage with [10].

\section{REFERENCES}

[1] K. Conradsen, A. A. Nielsen, and H. Skriver, "Determining the points of change in time series of polarimetric SAR data," IEEE Transactions on Geoscience and Remote Sensing, vol. 54, no. 5, pp. 3007-3024, May 2016, https://doi.org/10.1109/TGRS.2015.2510160.

[2] A. A. Nielsen, K. Conradsen, H. Skriver, and M. J. Canty, "Visualization of and software for omnibus test based change detected in a time series of polarimetric SAR data," Canadian Journal of Remote Sensing, vol. 43, no. 6, pp. 582-592, 2017, https://doi.org/10.1080/07038992.2017.1394182.

[3] K. Conradsen, A. A. Nielsen, J. Schou, and H. Skriver, "A test statistic in the complex Wishart distribution and its application to change detection in polarimetric SAR data," IEEE Transactions on Geoscience and Remote Sensing, vol. 41, no. 1, pp. 4-19, Jan. 2003, https://doi.org/10.1109/TGRS.2002.808066.

[4] M. J. Canty, Image Analysis, Classification and Change Detection in Remote Sensing, with Algorithms for Python, Taylor \& Francis, CRC Press, fourth revised edition, 2019.

[5] A. A. Nielsen, K. Conradsen, and H. Skriver, "Change detection in full and dual polarization, single- and multi-frequency SAR data," IEEE Journal of Selected Topics in Applied Earth Observations and Remote Sensing, vol. 8, no. 8, pp. 4041-4048, Aug. 2015, https://doi.org/10.1109/JSTARS.2015.2416434

[6] V. Akbari, S. N. Anfinsen, A. P. Doulgeris, T. Eltoft, G. Moser, and S. B. Serpico, "Polarimetric SAR change detection with the complex Hotelling-Lawley trace statistic," IEEE Transactions on Geoscience and Remote Sensing, vol. 54, no. 7, pp. 3953-3966, Jul. 2016, https://doi.org/10.1109/TGRS.2016.2532320.

[7] A. D. C. Nascimento, A. C. Frery, and R. J. Cintra, "Detecting changes in fully polarimetric SAR imagery with statistical information theory," IEEE Transactions on Geoscience and Remote Sensing, vol. 57, no. 3, pp. 1380-1392, Mar. 2019, https://doi.org/10.1109/TGRS.2018.2866367.

[8] S. K. Mitra, P. Bhimasankaram, and S. B. Malik, Matrix Partial Orders, Shorted Operators and Applications. Series in Algebra, vol. 10, World Scientific, Singapore, 2010.

[9] A. A. Nielsen, H. Skriver, and K. Conradsen, "The Loewner order and direction of detected change in Sentinel-1 and Radarsat-2 data," IEEE Geoscience and Remote Sensing Letters, vol. 17, no. 2, pp. 242-246, Feb. 2020, https://doi.org/10.1109/LGRS.2019.2918636.

[10] A. A. Nielsen, "Fast matrix based computation of eigenvalues and the Loewner order in polSAR data," IEEE Geoscience and Remote Sensing Letters, vol. 17, no. 10, pp. 1727-1731, Oct. 2020, https://doi.org/10.1109/LGRS.2019.2952202.

[11] J. J. van Zyl and F. T. Ulaby, "Scattering matrix representation for simple targets," in Radar Polarimetry for Geoscience Applications, F. T. Ulaby and C. Elachi, Eds. Artech, Norwood, MA, 1990.

[12] T. W. Anderson, An Introduction to Multivariate Statistical Analysis, John Wiley, New York, third edition, 2003.

[13] J. Schou, H. Skriver, A. A. Nielsen, and K. Conradsen, "CFAR edge detector for polarimetric SAR images," IEEE Transactions on Geoscience and Remote Sensing, vol. 41, no. 1, pp. 20-32, Jan. 2003, https://doi.org/10.1109/TGRS.2002.808063.

[14] S. N. Madsen, E. L. Christensen, N. Skou, and J. Dall, "The Danish SAR system: Design and initial tests," IEEE Transactions on Geoscience and Remote Sensing, vol. 29, pp. 417-426, 1991, https://doi.org/10.1109/36.79432.

[15] E. L. Christensen, N. Skou, J. Dall, K. Woelders, J. H. Jørgensen, J. Granholm, and S. N. Madsen, "EMISAR: An absolutely calibrated polarimetric L- and C-band SAR," IEEE Transactions on Geoscience and Remote Sensing, vol. 36, pp. 1852-1865, 1998, https://doi.org/10.1109/36.729356. 